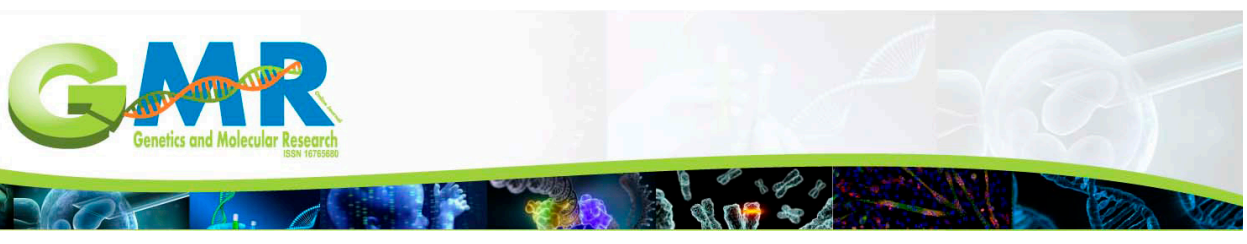

\title{
Phylogenetic analysis of Elymus (Poaceae) in western China
}

\author{
H. Song, Z.B. Nan and P. Tian \\ Key Laboratory of Grassland Agro-Ecosystems, \\ College of Pastoral Agriculture Science and Technology, \\ Lanzhou University, Lanzhou, China \\ Corresponding author: Z.B. Nan \\ E-mail: zhibiao@lzu.edu.cn
}

Genet. Mol. Res. 14 (4): 12228-12239 (2015)

Received February 4, 2015

Accepted June 26, 2015

Published October 9, 2015

DOI http://dx.doi.org/10.4238/2015.October.9.11

ABSTRACT. Elymus L. is often planted in temperate and subtropical regions as forage. Species in the genus have 5 allopolyploid genomes that are found in the grass tribe Triticeae. To determine the phylogenetic relationships in Elymus species from western China, we estimated phylogenetic trees using sequences from the nuclear ribosomal internal transcribed spacer and non-coding chloroplast DNA sequences from 56 accessions (871 samples) of 9 polyploid Elymus species and 42 accessions from GenBank. Tetraploid and hexaploid Elymus species from western China had independent origins, and Elymus species from the same area or neighboring geographic regions were the most closely related. Based on the phylogenetic tree topology, the St- and Y-genomes were not derived from the same donor and $Y$-genome likely originated from the H-genome of Hordeum species, or they shared the same origin or underwent introgression. The maternal genome of tetraploid and hexaploid Elymus species originated from species of Hordeum or Pseudoroegneria. Additionally, Elymus species in western China began diverging 178.5 million years ago, during a period of increased aridification as a consequence of the Messinian salinity crisis. Elymus species adapted to drought and high salinity may have developed based on the environmental 
conditions during this period. Elymus evolution in western China may have been affected by the uplift of the Qinghai-Tibetan Plateau (5 million years ago), when Elymus seeds were dispersed by gravity or wind into a newly heterogeneous habitat, resulting in isolation.

Key words: Elymus species; Evolution; ITS sequence; trnL-F sequence; Phylogeny; Poaceae

\section{INTRODUCTION}

Hybridization and polyploidization play important roles in plant evolution. Polyploidization, or chromosome doubling, can promote changes in genome size, cell size, genomic re-patterning, gene expression, retrotransposon activation, and epigenetic effects (Otto, 2007). Roughly 70\% of flowering plants are polyploids (Masterson, 1994) and there is evidence that polyploid species have a better chance of survival in unstable climates (Levin, 1983). Polyploidy can occur as autopolyploidy, by doubling a single genome, or allopolyploidy, by combining divergent genomes through hybridization (Masterson, 1994).

Elymus L. includes approximately 150 allopolyploid species in the grass tribe Triticeae and is the largest and most widely distributed genus in temperate and subtropic regions (Von Bothmer and Salomon, 1994). Notably, Elymus species have been widely examined in plant evolution. A chromosome pairing study found that Elymus species contains the St, H, P, W, and Y type genomes, potentially from the following donors: Pseudoroegneria, Hordeum, Agropyron, Australopyrum, and an unknown species, respectively (Torabinejad and Mueller, 1993). The St-genome is a shared genome found in all Elymus species (Dewey, 1984). Most Elymus species are either tetraploid StH and StY or they are hexaploid StStH, StHH, StSY, StYY, StHY, StYW, and StYP (Dewey, 1984). The StY tetraploid Elymus species are mainly distributed in Asia (Lu and Salömon, 1992).

Previous studies have described hypotheses for reticulate evolution estimated from molecular markers, including chloroplast DNA and high-copy and low-copy nuclear genes (MasonGamer et al., 2010b). Chloroplast DNA is maternally inherited in angiosperms, and generally there is a single copy of chloroplast loci present in plants. However, chloroplast DNA does not always clearly distinguish organismal phylogenetic relationships (Mason-Gamer, 2013). Low-copy nuclear genes can be inconvenient because they can be difficult to amplify because of their low-copy number, making it difficult to design amplification primers, particularly given that these regions have only been characterized in a few species (Mason-Gamer et al., 2010b). In contrast, the nuclear ribosomal internal transcribed spacer region (nrITS) is a high-copy gene that is easily cloned and is widely used for phylogenentic analyses. Distinct copies of the nrITS region may convert towards one or the other parent through a process of concerted evolution, even in polyploid genomes (MasonGamer, 2013), and thus some nrITS sequences may be discordant with the organismal phylogeny. Nevertheless, an analysis of numerous nrITS sequences from Elymus species confirmed that these sequences are suitable for estimating Elymus phylogenentic relationships (Liu et al., 2006).

Studies examining the phylogenetic relationships in Elymus species have generally used 2 approaches. Early studies examined hypotheses of phylogenetic relationships based on cytological data (Lu and Salömon, 1992). Subsequently, researchers focused on determining the origin of the Y-genome; currently, there are different views regarding the diploid donor of the Y-genome based on which molecular makers or whether the same Elymus species are sampled (Liu et al., 2006; 
Yan et al., 2011; Fan et al., 2013). Few molecular phylogenetic studies have included samples of Elymus species from western China.

The goals of the present study were to 1) elucidate the phylogenetic relationships of tetraploid and hexaploid Elymus species from western China; 2) reveal potential diploid, maternal donors using new and unreported chloroplast trnL-F sequences; and 3) estimate when Elymus species diverged in this region to test hypotheses regarding processes that may have led to speciation in the genus.

\section{MATERIAL AND METHODS}

\section{Plant materials and DNA extraction}

We collected 42 accessions (871 samples) of 9 polyploid Elymus species from western China from 2011-2013 and additional sequences from GenBank, including Elymus (StY-genome), Pseudoroegneria (St-genome), and Hordeum (H-genome) sequences. Bromus inermis was used as the outgroup (Liu et al., 2006) (Table S1).

We identified mature plants from each accession to confirm the species determination. Seeds were germinated in a greenhouse. Total genomic DNA was extracted from fresh leaf tissue using the cetyltrimethyl ammonium bromide method (Doyle and Doyle, 1987), with some modifications.

\section{DNA amplification and sequencing}

nrITS sequences were amplified by polymerase chain reaction using the primers ITS-5 (GGAAGTAAAAGTCGTAACAAGG) and ITS4 (TCCTCCGCTTATTGATGC) (Hsiao et al., 1995). Chloroplast non-coding trnL-F regions were amplified with rps (CGATAACGGGACATGAAG) and trnF (ATTTGAACTGGTGACACGAG) (Taberlet et al., 1991). Polymerase chain reaction was performed using a thermocycler protocol described in a previous report (Taberlet et al., 1991; Noyes and Rieseberg, 1999). Polymerase chain reaction products were cloned into the pMD 18-T vector (TaKaRa, Shiga, Japan), following the manufacturer instructions. Three to five positive colonies for each of the 56 accessions were sequenced by TaKaRa.

\section{Data analysis}

The nrITS sequences were aligned using the MAFFT 7.0 program (Katoh and Standley, 2013) and alignments were adjusted manually. Phylogenetic trees were estimated using maximum parsimony (MP) in PAUP 4.0b10 (Swofford, 2002). In MP analysis, a parsimony heuristic search used tree bisection-reconnection branch swapping and 100 random addition replicates. All characters were specified as unweighted, and the gaps were treated as missing data. Branch support for MP trees was used to assess the reliability of individual branches given the data and the model, based on 1000 bootstrap replicates.

nrITS dating analysis was implemented in BEAST 1.7.5 (Drummond and Rambaut, 2007). Because there are no reliable fossils of Elymus species that can be used as calibration points, we used a secondary calibration date of 29 (9-38) million years ago (MYA) based on a study that included well-described fossil calibrations (Bouchenak-Khelladi et al., 2010). The model GTR+G 
was used for dating analysis according to MrModeltest 2.3 (Drummond and Rambaut, 2007). The Markov chain Monte Carlo method was run for 40,000,000 generations, sampling every 4000 generations and discarding 1000 samples as burn-in. Parameter convergence and sample sizes were assessed using Tracer 1.4.

Median-joining (MJ) network analysis was applied to display the genealogical relationships between taxa (Jakob and Blattner, 2006; Fu and Allaby, 2010). Little information related to the phylogenetic network of Elymus species has been published. In the present study, we determined the haplotype of sequences, excluding the outgroup, using the DnaSP 5.0 program (Librado and Rozas, 2009) and constructed a network of the Elymus species using the Network 4.1 program (Fluxus Technology Ltd, Clare, Suffolk, UK). Recombination was tested using the Recco program (Maydt and Lengauer, 2006) and the MJ network was analyzed using non-recombining sequences.

\section{RESULTS AND DISCUSSION}

\section{nrlTS tree}

MP analysis yielded 239 equally parsimonious trees (tree length $=225$; consistency index $=0.8222$; retention index $=0.7379$ ). The strict consensus tree is shown in Figure 1. In the MP tree, clade I consisted of 2 subclades, $\mathrm{H} 1-2$. Subclade $\mathrm{H} 1$ contained 15 Elymus sequences, including 6 Elymus nutans, 4 Elymus excelsus, 3 Elymus tangutorum, and 2 Elymus dahuricus var. cylindricus. Six E. tangutorum were resolved in subclade $\mathrm{H} 2$. Additionally, 3 Elymus dolichatherus, 1 Elymus tibeticus, and 1 Pseudoroegneria strigosa sequences were not grouped in 1 subclade in clade I.

Clade II contained 25 accessions divided into 3 subclades. Subclade T1 included $2 E$. dahuricus, 2 E. dahuricus var. cylindricus, 1 E. tibeticus, 1 Elymus antiquus, and 1 E. tangutorum sequence. Five $E$. dolichatherus and $1 E$. antiquus sequences were resolved in subclade T2. Five Elymus sibiricus and 1 E. nutans sequences made up subclade T3. In addition, 3 Pseudoroegneria and 1 Elymus sequences were not grouped in 1 subclade in clade II. Furthermore, 4 Hordeum sequences with good bootstrap values were monophyletic in clade III.

The MP tree results showed that there were some single clades and low bootstrap value. This phenomenon was also observed in Elymus StH allotetraploids in a previous study (MasonGamer et al., 2010a). Clade I mainly included tetraploid Elymus species and clade II mainly contained hexaploid Elymus species (Figure 1). This indicates that tetraploid and hexaploid Elymus species have an independent origin.

Triticeae is widely distributed worldwide, and botanists have long been interested in how its species are related to one another. Early studies in Triticeae evolution focused on morphological characters, but it is difficult to recognize Triticeae species based on morphology. The development of molecular biology enabled to studies of the genetic diversity of Triticeae (Mason-Gamer et al., 2010b; Sha et al., 2010; Sun, 2014). Recent studies have focused on the origin and constitution of the Elymus genome (Sun, 2014). However, little is known regarding the genetic relationships between Elymus species in China.

Previously, most cytological studies of the StY-genome in Elymus species indicated that chromosome pairing in hybrids decreased if the hybrid parent was from a geographically distant location (Lu and Salömon, 1992). The Elymus species in clade I were mainly collected in Gansu Province and clade II contained Elymus species from the Qinghai-Tibetan Plateau, suggesting that there is a geographical component to the tree topology. The different allopolyploid types of 


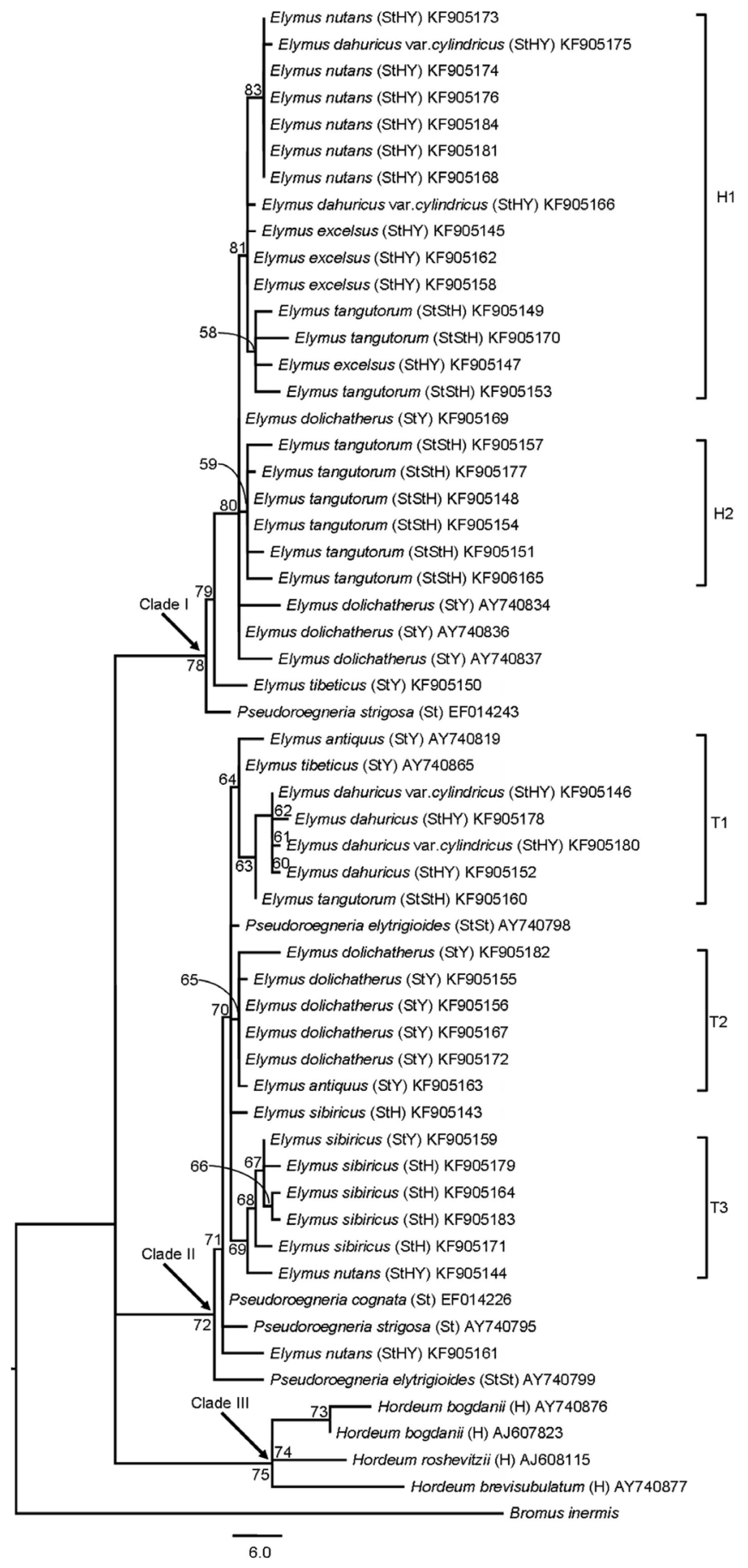

Figure 1. Maximum parsimony (MP) strict consensus tree based on nrITS sequences. Branches with bootstrap values $>50 \%$ are shown. Three major clades and many minor clades are described in the text. 
Elymus species may each represent distinct evolutionary lineages. For example, hexaploid Elymus species [except for E. dolichatherus (KF905169, AY740834, AY740836, and AY740837) and E. tibeticus (KF905150)] were in clade I, and tetraploid Elymus species (except for subclade T1) were in clade II. Species sharing similar morphological characters did not resolve as closely related in our trees. E. dolichatherus (AY740834, AY740836, and AY740837) from Sichuan Province and E. dolichatherus (KF905169) contained some nrITS copies in clade I, but collections (KF905155, KF905156, KF905167, and KF905172) from the Qinghai-Tibetan plateau were in clade II. Similar results were observed in Leymus based on nrlTS sequences (Sha et al., 2008). Their results showed that species from the same areas or neighboring geographic regions were closely related to each other Leymus species.

There have been several other studies examining the phylogenetic relationships between polyploid Elymus species and their putative diploid ancestors. North American Elymus allotetraploids include St- (Pseudoroegneria) and H (Hordeum)-genomic components (MasonGamer, 2001, 2013). Phylogenetic and cytogenetic analyses of Asian Elymus species showed that the putative parental genomes were from Pseudoroegneria (St-genome), Hordeum (H-genome), Agropyron (P-genome), Australopyrum (W-genome), and an unknown species (Y-genome) (Sun et al., 2008). However, in this study, Elymus species only contained St-, $\mathrm{H}$-, and Y-genomes based on cytological evidence.

Liu et al. (2006) suggested that the Elymus Y-genome evolved through gradual differentiation from Pseudoroegneria (St-genome). Our results on Elymus from western China do not support the results of Liu et al. (2006). Pseudoroegneria and the StY Elymus genome did not resolve as closely related (Figure 1). In addition, studies on the single copy nuclear $\beta$-amylase gene (Mason-Gamer et al., 2005), DNA polymerase subunit II (RPB2) sequences (Sun et al., 2008), and elongation factor $G(E F-G)$ sequence data (Yan et al., 2011) have suggested that the Elymus Y-genome originated in an independent $Y$ diploid species that is now extinct or undiscovered. However, Fan et al. (2013) suggested that the Elymus Y-genome is closely related to the Peridictyon sanctum (Xp -genome) based on single-copy nuclear acetyl-coa carboxylase-1 (Acc1) and phosphoglycerate kinase -1 (Pgk1) gene sequences. Therefore, the accumulated evidence suggests that the Elymus Y-genome originated in a different species or in multiple species. Unless a similar genome is identified in another grass, the donor of the Y-genome may never be identified (Mason-Gamer et al., 2010a; Mason-Gamer, 2013).

Interestingly, Hordeum species from the Xinjiang and Sichuan provinces in China are resolved in clade III, with no closely related Elymus species. This indicates that the Elymus $\mathrm{H}$-genome from western China could not have originated in diploid Hordeum species from Xinjiang and Sichuan provinces in China. Additionally, some studies (Helfgott and Mason-Gamer, 2004; Mason-Gamer, 2013) showed that North American Hordeum species were the diploid progenitors of the North American and Eurasian Elymus species. Other studies (Sun and Komatsuda, 2010; Fan et al., 2013) showed that the Hordeum H-genome from western China may have been a donor to Elymus, such as Hordeum bogdanii.

\section{Chloroplast DNA tree}

Parsimony analysis of trnL-F sequences in this study randomly yielded 317 equally parsimonious trees, with a tree length of 310 (consistency index $=0.9000$, rescaled consistency index $=0.7939$ ). The trnL-F phylogenetic relationships between Elymus and potential donor species, based on the MP method, are shown in Figure 2. 


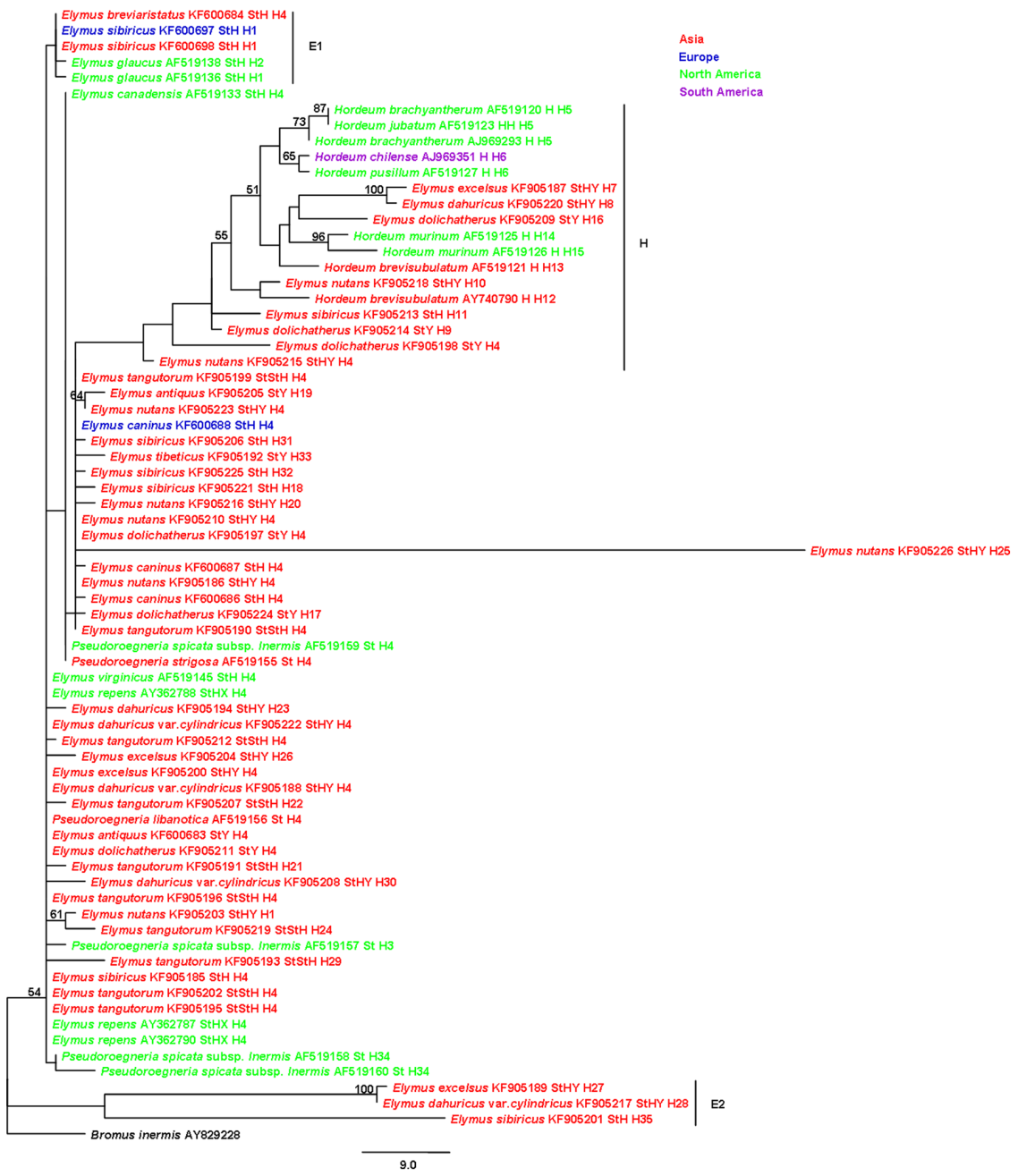

Figure 2. Strict consensus tree from maximum-parsimony (MP) analysis based on trnL-F sequences. Numbers with bootstrap values $>50 \%$ are shown. Three major clades and many minor clades are described in the text.

Compared with nuclear and chloroplast genes within phylogenetic relationships, the support provided by chloroplast genes is too weak to convincingly indicate actual polyphyletic origins (Mason-Gamer, 2013). However, chloroplast genes are inherited maternally, so the female parent of plants was recognized based on chloroplast gene sequence. In the present study, most bootstrap values on the MP phylogenetic trees were lower than $50 \%$, and only 3 clades were recognized, including clade E1, H, and E2 (Figure 2). Clade E1 was weakly supported and contained 1 Elymus breviaristatus (KF600684) and 1 E. sibiricus (KF600698) from Asia, 1 E. sibiricus (KF600697) from Europe, and 2 Elymus glaucus (AF519136 and AF519138) from North America. Clade E2 included 3 Asian Elymus with stronger support values, E. excelsus (KF905189), E. dahuricus var. cylindricus (KF905217), and E. sibiricus (KF905201). Notably, 8 Elymus species from Asia and 8 Hordeum species from the Americas ( 7 from North America and 1 from South America) were distributed 
in clade $\mathrm{H}$, with well-supported values. Our analysis of the phylogenetic relationships in clade $\mathrm{H}$ showed that E. excelsus (KF905187, StHY-genome), E. dahuricus (KF905220, StHY-genome), and E. dolichatherus (KF905209, StY-genome) clustered into a subclade with Hordeum murinum (AF519125 and AF519126, H-genome), while E. nutans (KF905218, StHY-genome) and Hordeum brevisubulatum (AY740790, H-genome) were grouped into another subclade. This indicated that either Hordeum is the maternal genome donor to Elymus species or that there was gene flow from Hordeum to Elymus species. Previous studies showed that the female parent of North American tetraploid Elymus species (StH-genome) originated from North American Pseudoroegneria species (Mason-Gamer and Orme, 2002; Sun, 2002), indicating that the female parent of Elymus species from North America and Asia have different origins. Interestingly, E. nutans (KF905218, StHYgenome) and $H$. brevisubutatum (AY740790, H-genome) species were from Asia, while Elymus species in the former group were from Asia, and Hordeum species were from North America and Asia. In this study, we found that Elymus species with the Y-genome may have originated from the Qing-Tibet Plateau approximately 17-8.5 MYA. In addition, Hordeum species originated in the Old World approximately 12 MYA (Blatter, 2006), and New World Hordeum species have persisted for at least 3-4 MYA (Jakob and Blattner, 2006). A major reason why Hordeum species could have dispersed between the Old and New Worlds is the emergence of the Bering Land Bridge during the Pleistocene.

Moreover, E. dolichatherus (KF905209, StY-genome) did not contain the H-genome, yet it clustered in a clade with Hordeum species. It remains unclear whether the $\mathrm{Y}$ - and $\mathrm{H}$ - genomes share the same origin. As described above, several hypotheses have been suggested to explain the origin of the Y-genome. Our results were not consistent with these hypotheses. However, our results indicated that the $\mathrm{Y}$ - and $\mathrm{H}$-genomes may have a common origin. However, Mason-Gamer (2013) suggested that the Y-genome of Asian Elymus does not fit into an existing genus, and instead may represent a single evolutionary lineage with subsequent introgression.

\section{Statistical MJ network}

Phylogenetic relationships reflect the hierarchical patterns of ancestry among different species, whereas networks are generally used to reflect ancestral and derived relationships (Posada and Crandall, 2001). To assess the maternal origins and biogeographical patterns of Elymus species, we constructed an MJ network of Elymus and its potential diploid donor (Figure $3)$. In the network, each circle indicated 1 haplotype, with the circle size proportional to the number of isolates with that haplotype, and median vectors indicating missing intermediates of unsampled nodes inferred by MJ network analysis. Different colors indicate different species; for example, yellow, gray, and green indicate Elymus, Horduem, and Pseudoroegneria species, respectively (Figure 3). The MJ network described the genealogical relationships among 35 haplotypes from 66 species and 7 missing intermediates (Figure 3). Among them, 5 haplotypes contained multiple species sequences: $\mathrm{H} 1$ included 5 Asian Elymus species; H4 included 26 Asian Elymus species, 2 Asian and 1 North American Pseudoroegneria species; H5 included 3 North American Hordeum species; H6 included 2 Hordeum species from North America and South America; and H34 included 2 North American Pseudoroegneria species. Other haplotypes included only 1 species (Table S1).

The network showed that Elymus species were grouped into 2 basic clusters of haplotypes, clusters 1 and 2. Compared to the trnL-F phylogenetic trees (Figure 2), cluster 2 containing Elymus and Hordeum species was consistent with clade $\mathrm{H}$, except for the presence of $E$. dolichatherus 
(KF905198) and E. nutans (KF905215) in phylogenetic trees. Therefore, the results of cluster 2 not only confirmed that the topology of the trnL-F phylogenetic trees was accurate, but also demonstrated that Elymus was the maternal donor to North American Hordeum species. Cluster 1 contained 20 polyloid Elymus haplotypes, 2 diploid Pseudoroegneria haplotypes, 1 nested haplotype, and 3 missing intermediates. Remarkably, Elymus and Pseudoroegneria species constituted the nested haplotype $\mathrm{H} 4$, indicating a contradiction between the results of trnL-F phylogenetic trees and the MJ network. In trnL-F phylogenetic analysis, Elymus and Pseudoroegneria species were not grouped into 1 clade; instead, most terminal branch lengths on phylogenetic trees were short. As described above, however, the MJ network described the genealogical relationship, and Mason-Gamer and Orme (2002) found that North American Pseudoroegneria species was the maternal genome donor to North American Elymus species. In conclusion, our results indicated that the Y-genome in Asian Elymus species likely had 2 patterns of maternal origin, with the first from Hordeum species and the second from Pseudoroegneria species.

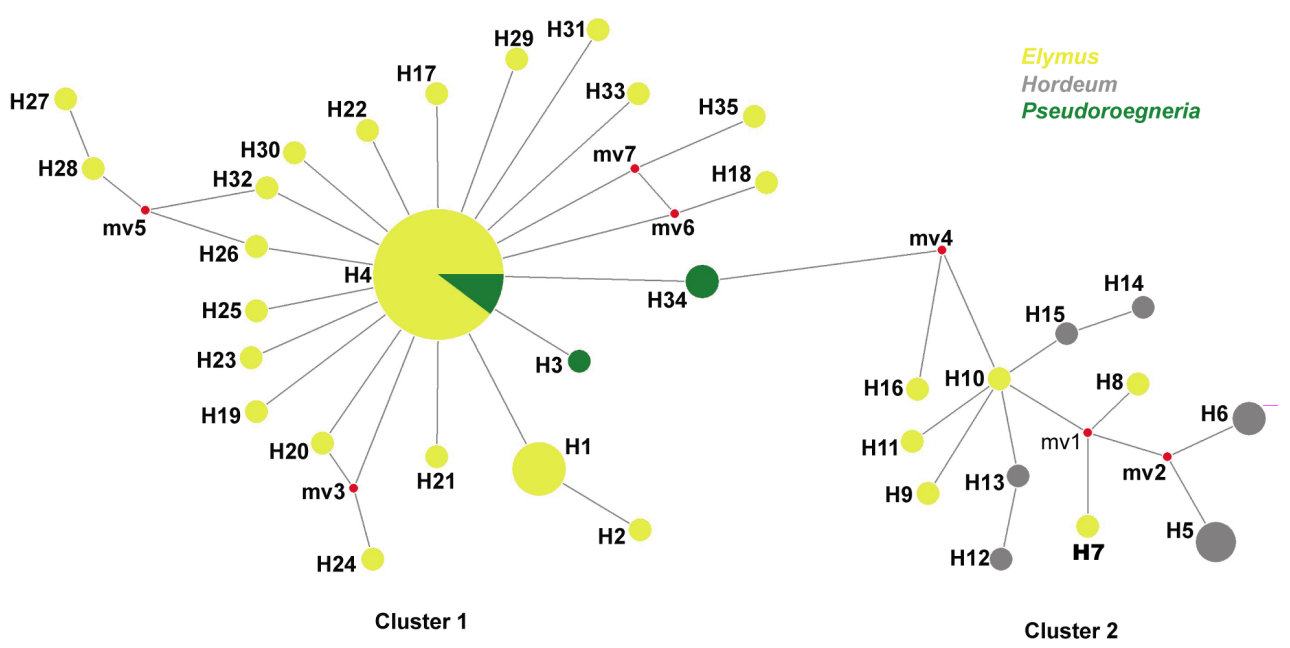

Figure 3. Median-joining (MJ) networks of trnL-F haplotypes from Elymus, Hordeum, and Pseudoroegneria species. Each circle indicated 1 haplotype, with circle size being proportional to the number of isolates with that haplotype, and median vectors indicating missing intermediates of unsampled nodes inferred by MJ network analysis. Yellow, gray, and green indicate Elymus, Horduem, and Pseudoroegneria species, respectively.

\section{Dating analysis}

Age estimates for the Elymus dataset found that the clades diverged 17-8.5 MYA. Sampled tetraploid Elymus species diverged approximately 17 MYA and sampled hexaploid Elymus species diverged approximately 8.5 MYA (Figure 4).

We estimated that Elymus species in western China diverged between 17-8.5 MYA. The Miocene (23-5.3 MYA) was a period of increasing aridification as a consequence of the Messinian salinity crisis (Wright and Cita, 1979). Elymus species may have adapted to drought and high salinity in the harsh environment during this period (Sasanuma et al., 2002). We hypothesize that selection for drought and salinity tolerance may have been the driving force for the divergence of Elymus species in western China, enabling them to withstand drought and high salinity. 


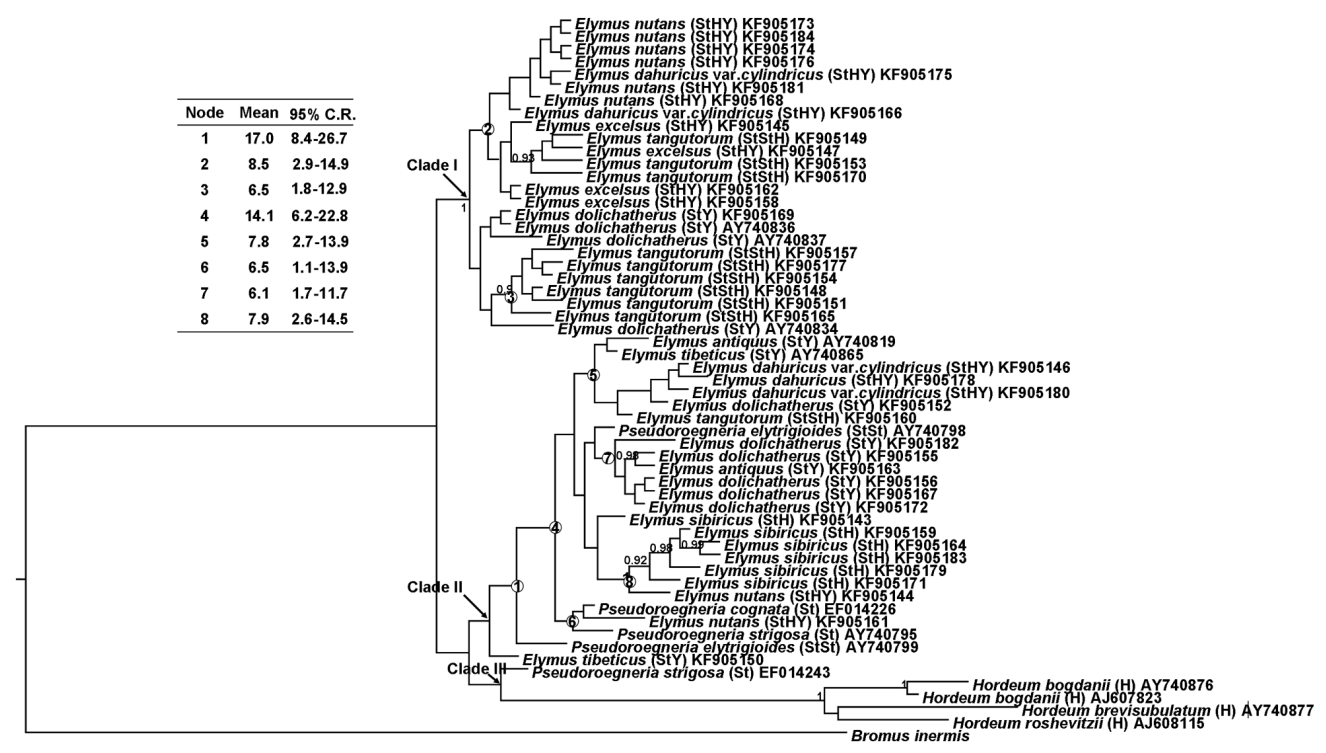

Figure 4. Bayesian analysis strict consensus tree based on nrlTS sequences. Branches with posterior probabilities $>0.9$ are shown. Table provides the estimated divergence dates for nodes $1-8$. Ages of nodes with $95 \%$ confidence ranges were obtained from BEAST molecular dating analysis. The $\mathrm{CR}$ ranges represent $95 \%$ highest posterior density values for nodes that received $>0.5$ posterior probabilities.

Central Asia is considered to be the center of origin of Elymus and the Y-genome is thought to have originated in Central Asia or the Himalayan region (Lu and Salömon, 1992; Torabinejad and Mueller, 1993). Geologic studies have shown that the Qinghai-Tibetan Plateau and adjacent areas were formed during large-scale uplifting in the Pliocene and during climate oscillations during the Quaternary (5 MYA) (Li and Pan, 2002). Nine Elymus species in this study are located mainly in Asia, particularly China (Lu and Salömon, 1992). Our dated tree indicated that the divergence times of these nodes (8) were more than 6 MYA (Figure 4), implying that these Elymus species already existed before uplifting of the Qinghai-Tibetan Plateau. Therefore, we suggest that 9 Elymus species in this study originated from the Qinghai-Tibetan Plateau. During the uplift of the Qinghai-Tibetan Plateau, Elymus seeds were dispersed by gravity or wind action into a newly heterogeneous habitat and experienced isolation.

\section{Conflicts of interests}

The authors declare no conflict of interest.

\section{ACKNOWLEDGMENTS}

Research supported by grants from the National Basic Research Program of China (\#2014CB138702). We are grateful to Xiuzhang Li, Gensheng Bao, Qiuyan Song, Xiang Yao, and Yong Liu for assistance in sampling. 


\section{Supplementary material}

\section{REFERENCES}

Blatter FR (2006). Multiple intercontinental dispersals shaped the distribution area of Hordeum (Poaceae). New Phytol. 169: 603-614.

Bouchenak-Khelladi Y, Verboom GA, Savolainen V and Hodgkinson T (2010). Biogeography of the grasses (Poaceae): a phylogenetic approach to reveal evolutionary history in geographical space and geological time. Bot. J. Linn. Soc. 162: 543-557.

Dewey DR (1984). The genomic system of classification. Aguide to intergeneric hybridization with the perennial Triticeae. In: Gene manipulation in Plant Improvement (Gustafson JP, eds.). Plenum Press, New York, 209-280.

Doyle JJ and Doyle JL (1987). A rapid DNA isolation procedure for small quantities of fresh leaf tissue. Phytochem. Bull. 19: 11-15.

Drummond AJ and Rambaut A (2007). BEAST: Bayesian evolutionary analysis by sampling trees. BMC Evol. Biol. 7: 214.

Fan X, Sha LN, Dong ZZ, Zhang HQ, et al. (2013). Phylogenetic relationships and Y genome origin in Elymus L. sensu lato (Triticeae; Poaceae) based on single-copy nuclear Acc1 and Pgk1 gene sequences. Mol. Phylogenet. Evol. 69: 919-928.

Fu YB and Allaby RG (2010). Phylogenetic network of Linum species as revealed by non-coding chloroplast DNA sequences. Genet. Resour. Crop Evol. 57: 667-677.

Helfgott DM and Mason-Gamer RJ (2004). The evolution of North American Elymus (Triticeae, Poaceae) allotetraploids: evidence from phosphoenolpyruvate carboxylase gene sequences. Syst. Bot. 29: 850-861.

Hsiao C, Chatterton NJ, Asay KH and Jensen KB (1995). Phylogenetic relationships of the monogenomic species of the wheat tribe, Triticeae (Poaceae), inferred from nuclear rDNA (internal transcribed spacer) sequences. Genome 38: 211-223.

Jakob SS and Blattner FR (2006). A chloroplast genealogy of Hordeum (Poaceae): long-term persisting haplotypes, incomplete lineage sorting, regional extinction, and the consequences for phylogenetic inference. Mol. Biol. Evol. 23: 1602-1612.

Katoh K and Standley DM (2013). MAFFT multiple sequence alignment software version 7: improvements in performance and usability. Mol. Biol. Evol. 30: 772-780.

Levin D (1983). Polyploidy and novelty in flowering plants. Am. Nat. 122: 1-25.

Li BY and Pan BT (2002). Progress in paleogeographic study of the Tibetan Plateau. Geogr. Res. 21: 61-70.

Librado P and Rozas J (2009). DnaSP v5: a software for comprehensive analysis of DNA polymorphism data. Bioinformatics 25: 1451-1452.

Liu Q, Ge S, Tang H, Zhang X, et al. (2006). Phylogenetic relationships in Elymus (Poaceae, Triticeae) based on the nuclear ribosomal transcribed spacer and chloroplast trnL-F sequences. New Phytol. 170: 411-420.

Lu BR and Salömon B (1992). Differentiation of the SY genomes in Asiatic Elymus. Hereditas 116: 121-126.

Mason-Gamer RJ (2001). Origin of North American Elymus (Poaceae: Triticeae) allotetraploids based on granule-bound starch synthase gene sequences. Syst. Bot. 26: 757-758.

Mason-Gamer RJ (2013). Phylogeny of a genomically diverse group of Elymus (Poaceae) allopolyploids reveals multiple levels of reticulation. PLoS One 8: e78449.

Mason-Gamer RJ and Orme NL (2002). Phylogenetic analysis of North American Elymus and the monogenomic Triticeae (Poaceae) using three choroplast DNA data set. Genome 45: 991-1002.

Mason-Gamer RJ, Burns MM and Naum M (2005). Polyploidy, introgression, and complex phylogenetic patterns within Elymus. Czech. J. Genet. Plant. 41: 21-26.

Mason-Gamer RJ, Burns MM and Naum M (2010a). Phylogenetic relationships and reticulation among Asian Elymus (Poaceae) allotetraploids: analyses of three nuclear gene trees. Mol. Phylogenet. Evol. 54: 10-22.

Mason-Gamer RJ, Burns MM and Naum M (2010b). Reticulate evolutionary history of a complex group of grasses: phylogeny of Elymus StStHH allotetraploids based on three nuclear genes. PLoS One 5: e10989.

Masterson J (1994). Stomatal size in fossil plants: evidence for polyploidy in majority of angiosperms. Science 264: 421-424.

Maydt J and Lengauer T (2006). Recco: recombination analysis using cost optimization. Bioinformatics 22: 1064-1071.

Noyes RD and Rieseberg LH (1999). ITS sequence data support a single origin for North American Astereae (Asteraceae) and reflect deep geographic divisions in Aster S.L. Am. J. Bot. 86: 398-412.

Otto SP (2007). The evolutionary consequences of polyploidy. Cell 131: 452-462.

Posada D and Crandall KA (2001). Intraspecific gene genealogies: trees grafting into networks. Trends Ecol. Evol. 16: $37-45$.

Sasanuma T, Endo TR and Ban T (2002). Genetic diversity of three Elymus species indigenous to Japan and East Asia ( $E$. tsukushiensis, E. humidus and E. dahuricus) detected by AFLP. Genes Genet. Syst. 77: 429-438.

Sha L, Yang R, Fan X, Wang X, et al. (2008). Phylogenetic analysis of Leymus (Poaceae: Triticeae) inferred from nuclear rDNA ITS sequences. Biochem. Genet. 46: 605-619. 
Sha L, Fan X, Yang R, Kang H, et al. (2010). Phylogenetic relationships between Hystrix and its closely related genera (Triticeae; Poaceae) based on nuclear Acc1, DMC1 and chloroplast trnL-F sequences. Mol. Phylogenet. Evol. 54: 327-335.

Sun $G$ (2002). Interspecific polymorphism at non-coding regions of chloroplast, mitochondrial DNA and rRNA IGS region in Elymus species. Hereditas 137: 119-124.

Sun G (2014). Molecular phylogeny revealed complex evolutionary process in Elymus species. J. Syst. Evol. 9999: 1-6.

Sun $G$ and Komatsuda T (2010). Origin of the $Y$ genome in Elymus and its relationship to other genomes in Triticeae based on evidence from elongation factor G (EF-G) gene sequences. Mol. Phylogenet. Evol. 56: 727-733.

Sun GL, Ni Y and Daley T (2008). Molecular phylogeny of RPB2 gene reveals multiple origins, geographic differentiation of $\mathrm{H}$ genome, and the relationship of the $\mathrm{Y}$ genome to other genomes in Elymus species. Mol. Phylogenet. Evol. 46: 897-907.

Swofford DL (2002). PAUP*: Phylogenetic analysis using parsimony (*and Other Methods), version 4b10. Sinauer, Sunderland.

Taberlet P, Gielly L, Pautou $G$ and Bouvet J (1991). Universal primers for amplification of three non-coding regions of chloroplast DNA. Plant Mol. Biol. 17: 1105-1109.

Torabinejad J and Mueller RJ (1993). Genome constitution of the Australian hexaploid grass Elymus scabrus (Poaceae: Triticeae). Genome 36: 147-151.

Von Bothmer R and Salomon B (1994). Triticeae: a tribe for food, feed and fun. In: Proceedings of the 2nd International Triticeae Symposium (Wang RRC, Jensen KB and Jaussi C, eds.). Utah State University Press, Utah, 1-12.

Wright R and Cita MB (1979). Geo- and biodynamic effects of the Messinian salinity crisis in the Mediterranean. Palaeogeogr. Palaeocl. 29: 215-222.

Yan C, Sun G and Sun D (2011). Distinct origin of the $Y$ and St genome in Elymus species: evidence from the analysis of a large sample of St genome species using two nuclear genes. PLoS One 6: e26853. 